\title{
The Usefulness of an Oral-Health Promotion Program on Japanese Working-Age Population: A Pilot Study.
}

\author{
Yukihide Aoyama ${ }^{1}$, Terumitsu Murakami ${ }^{2}$, Chiyoko Uchiyama ${ }^{1}$, Yoshihiro Tamura ${ }^{3}$, Toshiaki Oyama ${ }^{3}$, \\ Itoyo Tokuda ${ }^{4}, K^{2}$ iji Wada ${ }^{4}$, Yukio Yamamoto ${ }^{1}$, Wataru Kobayashi ${ }^{3}$, Kazushige Ihara $^{4}$, Koichi Murashita ${ }^{5}$, \\ Shigeyuki Nakaji ${ }^{4,5}$ \\ ${ }^{1}$ Advanced Oral Health Science Research Laboratories, Research and Development Headquarters, LION Corporation, \\ Edogawa-ku, Japan \\ ${ }^{2}$ DX Promotion Department, LION Corporation, Sumida-ku, Japan \\ ${ }^{3}$ Department of Oral and Maxillofacial Surgery, Graduate School of Medicine, Hirosaki University, Hirosaki, Japan \\ ${ }^{4}$ Department of Social Medicine, Graduate School of Medicine, Hirosaki University, Hirosaki, Japan \\ ${ }^{5}$ COI Research Initiatives Organization, Hirosaki, Japan
}

Objectives: Oral health is an important factor contributing to work performance, productivity, and general health of workers. Nevertheless, oral-health promotion is frequently neglected within occupational health, because dental examination is costly and time-consuming. Therefore, we developed an Oral-Health Promotion Program (OHPP), which is easy-to-implement without dentists, intending to achieve the oral-health promotion even at the workplace. This study aimed to examine the usefulness of the OHPP on Japanese working population.

Methods: The OHPP was conducted for 65 employees (39 men and 26 women) working in a company in Hirosaki city, Japan. At baseline, all subjects underwent salivary multi test (SMT) and responded to questionnaires regarding to their oral hygiene behaviors or oral-health conditions. On the same day, they received feedback and oral-health education by dental hygienists, based on the SMT or the questionnaires. Subsequently, 7 months follow-up was performed, and the subjects had received oral-health information during the follow-up. After the follow-up, the SMT and the questionnaires were performed on the same subjects again, and oral hygiene behaviors or oral-health conditions were compared to those at the baseline. To assess their oral-health conditions adequately, dental examination was also conducted by dentists at the baseline and after the follow-up.

Results: Compared to the baseline, frequencies of tooth brushing were significantly increased $(p<0.05)$ after the follow-up. The dental examination by dentists revealed that the prevalence of gingivitis (bleeding on probing) was also significantly decreased between the baseline and after the follow-up $(\mathrm{p}<0.05)$. Furthermore, the improvement of tooth-brushing frequencies and the prevalence of gingivitis were significantly associated $(\mathrm{p}<0.05)$.

Conclusions: Our results demonstrated that the OHPP, which is easily implemented, can be effective in improving oral hygiene behaviors, and consequently oral-health conditions of working-age population in Japan.

Keywords: Oral health promotion, pilot study, salivary multi test

Copyright (C) 2021. Korean Academy of Preventive Dentistry. All rights reserved.

This is an Open Access article distributed under the terms of the Creative Commons Attribution Non-Commercial License (http://creativecommons.org/licenses/ by-nc/4.0) which permits unrestricted non-commercial use, distribution, and reproduction in any medium, provided the original work is properly cited. 